\title{
Spatial-temporal patterns of malaria incidence in Uganda using HMIS data from 2015 to 2019
}

Simon P. Kigozi ${ }^{1,2^{*}} \mathbb{D}$, Ruth N. Kigozi ${ }^{3}$, Catherine M. Sebuguzi ${ }^{2,4}$, Jorge Cano ${ }^{1}$, Damian Rutazaana ${ }^{4}$, Jimmy Opigo ${ }^{4}$, Teun Bousema ${ }^{5}$, Adoke Yeka ${ }^{6}$, Anne Gasasira ${ }^{7}$, Benn Sartorius ${ }^{1}$ and Rachel L. Pullan ${ }^{1}$

\begin{abstract}
Background: As global progress to reduce malaria transmission continues, it is increasingly important to track changes in malaria incidence rather than prevalence. Risk estimates for Africa have largely underutilized available health management information systems (HMIS) data to monitor trends. This study uses national HMIS data, together with environmental and geographical data, to assess spatial-temporal patterns of malaria incidence at facility catchment level in Uganda, over a recent 5-year period.
\end{abstract}

Methods: Data reported by 3446 health facilities in Uganda, between July 2015 and September 2019, was analysed. To assess the geographic accessibility of the health facilities network, AccessMod was employed to determine a three-hour cost-distance catchment around each facility. Using confirmed malaria cases and total catchment population by facility, an ecological Bayesian conditional autoregressive spatial-temporal Poisson model was fitted to generate monthly posterior incidence rate estimates, adjusted for caregiver education, rainfall, land surface temperature, night-time light (an indicator of urbanicity), and vegetation index.

Results: An estimated 38.8 million (95\% Credible Interval [Cl]: 37.9-40.9) confirmed cases of malaria occurred over the period, with a national mean monthly incidence rate of 20.4 (95\% Cl: 19.9-21.5) cases per 1000, ranging from 8.9 (95\% Cl: 8.7-9.4) to 36.6 (95\% Cl: 35.7-38.5) across the study period. Strong seasonality was observed, with June-July experiencing highest peaks and February-March the lowest peaks. There was also considerable geographic heterogeneity in incidence, with health facility catchment relative risk during peak transmission months ranging from 0 to 50.5 (95\% Cl: 49.0-50.8) times higher than national average. Both districts and health facility catchments showed significant positive spatial autocorrelation; health facility catchments had global Moran's I = 0.3 $(p<0.001)$ and districts Moran's I = $0.4(p<0.001)$. Notably, significant clusters of high-risk health facility catchments were concentrated in Acholi, West Nile, Karamoja, and East Central - Busoga regions.

(Continued on next page)

\footnotetext{
* Correspondence: skigozi@yahoo.com

'Department of Disease Control, London School of Hygiene \& Tropical

Medicine, Keppel Street, London WC1E 7HT, UK

Infectious Diseases Research Collaboration, PO Box 7475, Kampala, Uganda

Full list of author information is available at the end of the article
}

(C) The Author(s). 2020 Open Access This article is licensed under a Creative Commons Attribution 4.0 International License, which permits use, sharing, adaptation, distribution and reproduction in any medium or format, as long as you give appropriate credit to the original author(s) and the source, provide a link to the Creative Commons licence, and indicate if changes were made. The images or other third party material in this article are included in the article's Creative Commons licence, unless indicated otherwise in a credit line to the material. If material is not included in the article's Creative Commons licence and your intended use is not permitted by statutory regulation or exceeds the permitted use, you will need to obtain permission directly from the copyright holder. To view a copy of this licence, visit http://creativecommons.org/licenses/by/4.0/. The Creative Commons Public Domain Dedication waiver (http://creativecommons.org/publicdomain/zero/1.0/) applies to the data made available in this article, unless otherwise stated in a credit line to the data. 
(Continued from previous page)

Conclusion: Findings showed clear countrywide spatial-temporal patterns with clustering of malaria risk across districts and health facility catchments within high risk regions, which can facilitate targeting of interventions to those areas at highest risk. Moreover, despite high and perennial transmission, seasonality for malaria incidence highlights the potential for optimal and timely implementation of targeted interventions.

Keywords: Uganda, Malaria, Incidence, Relative risk, Routine surveillance, HMIS, Seasonality

\section{Background}

The global burden of malaria has declined since 2000 primarily due to the scale-up of control interventions, including long-lasting insecticidal nets (LLINs), indoor residual spraying with insecticide (IRS), and use of artemisinin-based combination therapy (ACT) [1-3]. Nevertheless, incidence rates in sub-Saharan Africa remained high at an estimated 219 cases per 1000 in 2017-2018 [3]. The incidence estimates used to monitor trends across sub-Saharan Africa are typically generated using parasite prevalence in children $2-10$ years fitted in prevalence-to-incidence models [3]. Though informative, the surveys included happen infrequently [4] and may be limited in scale. Derived burden estimates, therefore, cannot adequately support day-to-day monitoring for decision making at national or sub-national levels [5].

National malaria control programmes typically depend on routine health management information systems (HMIS) data to guide programme decisions in control and elimination efforts. With the advent and extended access to web-based health information systems, such as the District Health Information System - version 2 (DHIS-2), timely access to nation-wide HMIS data and quality of these data have been shown to have greatly improved in sub-Saharan Africa [6, 7]. As such, the WHO has reiterated that timely and high-quality HMISbased burden estimates are achievable, and can be used to inform on-going decision making [8]. Despite this, HMIS remains underutilized, especially for risk mapping, due to concerns over incompleteness and delayed reporting $[3,9,10]$. Whilst HMIS has had, and still needs, improvement, substantial discrepancies between estimates of malaria burden from the current prevalence-to-incidence model approach and HMISbased reports persist among at least 30 high burden countries [3]. Thus, questions remain as to the reliability of HMIS-based estimates and their corresponding representation of fine-scale spatial distribution of risk to support evidence-based decision making by country-level programme managers.

Small area space-time disease models fitted to routinely reported data have been widely implemented to accurately identify contextually important risk factors and unpack spatial-temporal patterns of infectious diseases, including tuberculosis and malaria [11-15]. These models have the capacity to explain the spatial autocorrelation in disease data, and can provide robust means of understanding ecological connectivity and relationships [16] that are critical for control processes in high malaria or other disease burden countries. Moreover, foci of high malaria risk or burden are pertinent to the principle of strategic information to drive impact under the global high burden to high impact initiative, for effective targeting of interventions [17]. This study therefore, aims to investigate a pragmatic novel small-area space-time approach using a nationwide network of health facilities in estimating malaria incidence from HMIS data, in order to identify areas of high malaria burden and risk across Uganda and assess malaria seasonality.

\section{Methods}

\section{Summary}

In brief, the study applied a Bayesian space-time Poisson regression model to assess the spatiotemporal variability of incidence of confirmed malaria (as reported through the national HMIS) at a fine spatial scale (health facility catchment, 3446 catchments with contiguous neighbours ranging from 0 to 11 (Fig. S16, Additional file 1)) by month (July-2015 to September-2019, 51 months), including primary caregiver education, rainfall, land surface temperature, night-time light, vegetation index, and spatial random effects to account for inherent correlation. To do this, health facility catchment cartographies and demographics were developed using multiple sources as described below.

\section{Study setting}

Uganda was estimated to be the 3rd highest contributor of Plasmodium falciparum malaria cases globally in 2018, with incidence rates of $>250$ cases per 1000 population at risk within a perennial transmission setting [18]. Located between $-1^{0}$ and $4^{0}$ latitudes, it covers a total area of $\approx 241,500 \mathrm{~km}^{2}$ that was divided into 15 nonadministrative regions (comprised of between one to 13 districts each) considered to be the malaria endemicity zones under the Uganda Demographic and Health Survey (UDHS) Program by 2018 [19]. Nested within these regions were 128 districts (as they were known in 2018), representing the second administrative level of government. 


\section{Data and population}

\section{Health management information systems data}

In Uganda, all health facilities are required to submit monthly reports from their out-patients department (OPD) registers on all reported diseases to the Department of Health information of the Ministry of Health $(\mathrm{MoH})$. Health facilities are either private-for-profit (PFP) or public comprised of the government owned and private-not-for-profit (PNFP) facilities. HMIS was introduced in 1997 as a paper-based reporting system from each health facility to the Ministry of Health. In 2012, however, a web-based reporting version, the DHIS-2, was implemented with full roll-out across the country in 2013 [20]. In this system, health facility data was either entered directly among high-level facilities or sent as paper reports from lower-level facilities to the districts for entry into the online system.

For this study, HMIS data consisted of monthly counts of all reported and confirmed malaria cases from study facilities, defined here as reporting facilities with available geo-coordinates. Reported malaria cases were defined as all cases reported, regardless of confirmation status, while confirmed malaria were laboratory confirmed cases using either blood slide microscopy (B/ S) or rapid diagnostic test for malaria (RDT) - per national guidelines. Whereas the recruited reporting facilities with available geo-coordinates represented 3453/ 7029 (49.1\%) of all facilities included within the DHIS-2, 2656/7029 (37.8\%) neither reported nor were geolocated and were therefore not recruited (Fig. S1, Additional file 1). Whilst majority of reporting geolocated facilities were publicly owned, the majority of nongeolocated health facilities were private for profit (PFP) commonly located in urban areas and these were excluded. Notably, the two districts of Kampala and Wakiso that together formerly comprised the capital city, contributed $49 \%$ of these excluded facilities (Fig. S4, Additional file 1). All reporting facilities that were not geolocated or geolocated facilities without a matching reporting health facility were excluded from this study. A total of 3446 geo-located health facilities constituted the study facilities for this work (Fig. 1).

\section{Ancillary data}

To define accessibility to health facilities, four categories of single timepoint ancillary data were incorporated to develop a cost-distance surface (Table 1). First, a digital elevation model (DEM) provided a measure of penalty on travel speed depending on direction of travel along the elevation. Second, a land use and land cover raster data set from 2016 was used to define diversity of land cover across which, travel speed would be affected. Third, wetlands, lakes, and rivers were identified as barriers for travel. Lastly, road networks were incorporated and categorized by feasible travel speed class.

To generate predicted incidence rates, accounting for spatially variable risk factors, ancillary data sets at multitime points were considered and utilized (Table 1). Notably, whilst vegetation quantities (NDVI) were quantified as the first 10 days (dekad) per month and rainfall as monthly estimates, monthly night-light emissivity was projected using 2012 and 2016 data sets, and the mean number of years of attending school among childbearing women published in [21] were included as a single estimate.

\section{Health facility catchments}

Currently, the HMIS is used to report malaria burden down to the district level, limiting the ability to observe and act upon heterogeneity at finer spatial scales. In part, this is because of limited information on health facility catchments. Considering proximity as the most important determinant of health facility access and utility [22, 23], health facility catchments were defined based on a cost-distance surface generated using a WHO supported tool known as AccessMod [24] as described in (Section D, Additional file 1). This tool has been widely used in assessments for general and emergency care accessibility, and the estimation of care utilization for febrile illnesses, among others [25-27].

Using the cost-distance surface generated based on anisotropic (direction dependent) analysis, with direction of travel considered as 'towards the health facility' in the geographic accessibility model, three-hour travel catchment buffers were generated for each health facility included in the study, given the distribution of travel time (Fig. S5, Additional file 1). To delineate each facility's catchment area, the intersection polygon between the three-hour travel buffer and a Thiessen polygon around each health facility, generated using ESRI ArcGIS 10.5 Thiessen polygon tool (ESRI 1995-2016; Redlands, CA, USA), was derived. This intersection polygon constituted the catchment area for each health facility covering majority of the country.

\section{Population data}

Population estimates for the country were obtained from gridded population surfaces generated by the WorldPop project whose estimates are based on national census estimates and other factors, accessible from www.worldpop.org. Annual gridded population surfaces were obtained for the duration between 2014 and 2019 and population estimates per year extracted as summary statistics for each calendar year of the study duration 2015 to 2019. These estimates were extracted using ESRI ArcGIS 10.5 Zonal Statistics tool at the level of the defined catchment area for each 


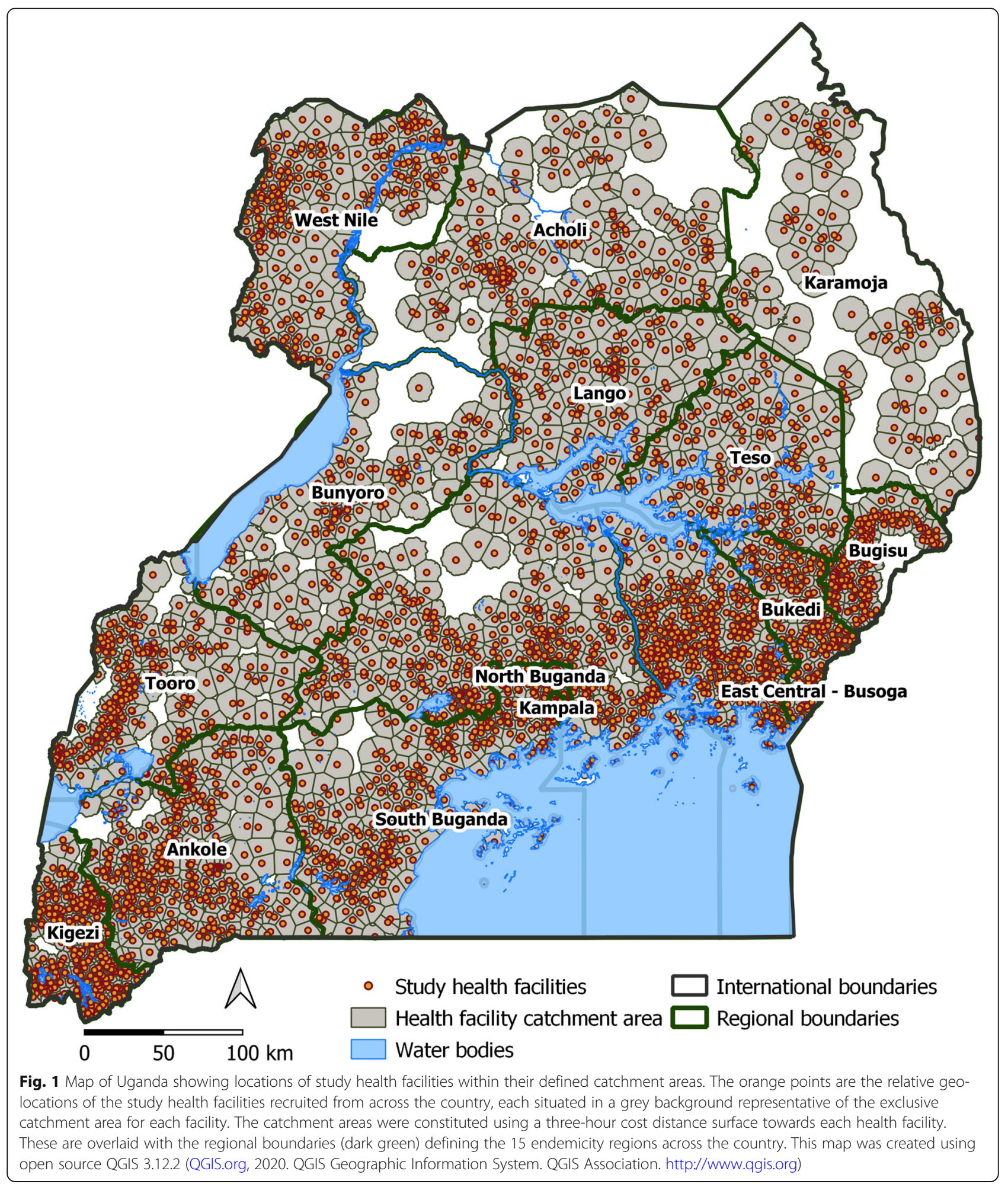


Table 1 Description of ancillary data sets and the sources of these covariates

\begin{tabular}{|c|c|c|}
\hline Data set & $\begin{array}{l}\text { Data } \\
\text { type }\end{array}$ & Data source \\
\hline \multicolumn{3}{|l|}{ Single time point data sets } \\
\hline National geo-located health facilities & Vector & $\begin{array}{l}\text { https://figshare.com/articles/Public_health_facilities_in_sub_Saharan_Africa/7725374 } \\
\text { Accessed September-2019. }\end{array}$ \\
\hline Digital elevation model & Raster & https://www.rcmrd.org/ Accessed October-2019. \\
\hline Land use and land cover & Raster & $\begin{array}{l}\text { http://geoportal.rcmrd.org/layers/servir\%3Auganda_sentinel2_lulc2016 Accessed } \\
\text { October-2019. }\end{array}$ \\
\hline National wetlands & Vector & $\begin{array}{l}\text { http://maps.nema.go.ug/layers/geonode\%3Augandawetlands2008 Accessed } \\
\text { September-2019. }\end{array}$ \\
\hline Lakes and rivers & Vector & $\begin{array}{l}\text { https://geodata.lib.berkeley.edu/catalog/stanford-fh022bz4757 Accessed } \\
\text { September-2019. }\end{array}$ \\
\hline Road network & Vector & $\begin{array}{l}\text { http://cod.humanitarianresponse.info/sites/default/files/uganda_roads_feb2009.zip } \\
\text { Accessed September-2019 and from KEMRI. }\end{array}$ \\
\hline \multicolumn{3}{|l|}{ Multi-time point data sets } \\
\hline Land surface temperature & Raster & \multirow{2}{*}{$\begin{array}{l}\text { https://earlywarning.usgs.gov/fews/ewx/index.html?region=af Accessed } \\
\text { October-2019. }\end{array}$} \\
\hline $\begin{array}{l}\text { Normalized difference vegetation } \\
\text { index (NDVI) }\end{array}$ & Raster & \\
\hline Rainfall & Raster & https://www.tamsat.org.uk/data/archive Accessed September-2019. \\
\hline Night-light emissivity & Raster & https://earthobservatory.nasa.gov/features/NightLights Accessed November-2019. \\
\hline $\begin{array}{l}\text { Mean years of education for women of } \\
\text { childbearing age over } 2000-2015\end{array}$ & Raster & $\begin{array}{l}\text { http://ghdx.healthdata.org/record/africa-educational-attainment-geospatial-estimates-2000-2015 } \\
\text { Accessed November-2019. }\end{array}$ \\
\hline
\end{tabular}

study health facility, regardless of administrative boundaries, given that care seeking is not restricted by these boundaries in Uganda.

\section{Spatial, temporal, and spatial-temporal analyses}

The primary outcome in this analysis was monthly malaria incidence rate, derived from HMIS data as the number of new confirmed cases per facility catchment divided by the total population of the catchment per month (a proxy for person-time).

Inherent spatial correlation of malaria infections is unexplained within classical regression approaches though remains in the residuals and induces spatial autocorrelation in the response even after known available risk factors are accounted for [28]. Using conditional autoregressive models, however, explains this autocorrelation in the outcome using random effects within a Bayesian framework that uses prior distribution, maximum likelihood, and neighbourhood predicts a more reliable outcome $[29,30]$. As such, a Bayesian space-time model employing BYM (Besag, York and Mollie) conditional autoregressive random effects and using integrated nested Laplace approximation (INLA) (www.r-inla.org), was fitted to the monthly crude confirmed case rates in $\mathrm{R}$ [31]. The model included structured and unstructured spatial effects, as well as structured and unstructured temporal effects, to explain measured (structured) and unmeasured (unstructured) risk factor impacts on the posterior estimates of incidence. The unobserved spatial correlation in the form of noise, attributable to independent health facility catchments, was accounted for through random effects. This model can be summarized as $=\boldsymbol{\mu}+\boldsymbol{\beta} \boldsymbol{x}_{\boldsymbol{i}}+\boldsymbol{b}_{\boldsymbol{i}}+\boldsymbol{c}_{\boldsymbol{i}}$, with $y$ denoting the posterior estimates of incidence rates, $\mu$ the crude incidence rates (correlated with the posterior estimates (Fig. S6 and Fig. S7, Additional file 1)), $x_{i}$ the covariates estimating the risk factors, $b_{i}$ and $c_{i}$ the overall spatial and temporal random effects respectively, that are also determined conditional on random effects of neighbouring catchments [32]. Also, to avoid overfitting, a time restriction using a random walk of the first order was included.

Candidate covariates had been used in other studies, given their association with malaria transmission, including rainfall, temperature, vegetation index, night-time lights (a proxy for urbanicity), and caregiver education [13, 33-35]. For inclusion in the final model, covariates quantities were evaluated for impact on a linear regression model of crude incidence rates, considering lower Akaike's information criteria values (Table S2, Additional file 1). The final covariate list included catchments estimates of mean years of education for women of childbearing age, mean of current and 3 months' lags for both rainfall and land surface temperature estimates, mean monthly night-time light emissivity, and mean of current and 1 month's lag of vegetation amounts. All these were significantly associated with crude incidence estimates (Table S3, Additional file 1). Both $\beta$ and $b$ 
were assigned monthly informative Gaussian distributions over the full 51 months length of the study duration. The full model was validated by withholding $20 \%$ of data points at random and comparing the model predicted values with the actual observed values using scatter plots and spearman's correlation coefficients (Section F, Additional file 1).

The relative risk of malaria at district and health facility catchment levels was derived as the respective predicted incidence rate divided by the overall predicted mean incidence rate at the national level per calendar month of the study duration. All maps of the posterior estimates of incidence rates and relative risk of malaria were generated using R ( $\mathrm{R}$ Foundation for Statistical Computing, Vienna, Austria).

Spatial clustering in the modelled outcome was further investigated using the global Moran's Index statistic within the spatial dependence (spdep) package of R. This was coupled with visual examination of Moran's scatter plots of incidence and risk estimates, at both district and health facility catchment resolutions. To identify cluster locations, the local Moran's Index using ESRI ArcGIS 10.5 Cluster and Outlier Analysis (Anselin Local Moran's I) tool was used, set for first order queen contiguity, running 999 permutations and clusters evaluated at 0.01 level of significance.

Also, study model estimates of confirmed malaria cases were compared with estimates from both the WHO's recent reports $[3,18,36]$ and Malaria Atlas Project (MAP) estimates for the same period from https://malariaatlas. org/trends/country/UGA (Section I, Additional file 1) and relationship between MIS regional prevalence estimates [19] and estimated relative risk examined using visual inspection of scatter-plots (Fig. S12, Additional file 1).

\section{Results}

\section{Study population}

The total population identified within the health facility catchments, considered at risk of malaria infection and likely to seek care from the associated geo-located publicly reporting health facility, were considered the study population of interest. The total population was estimated at 34.9 and 39.6 million in 2015 and 2019, respectively, with $\approx 2.8 \%$ of the population located outside of the defined catchments (Section C, Additional file 1).

\section{HMIS data summary}

Between 62.2 and $88.7 \%$ of nationally reported cases of malaria annually were diagnostically confirmed cases in 2015 and 2019, respectively (Fig. S2, Additional file 1). Whilst these proportions increased across the 15 regions of the country over time, Kampala recorded marginal improvements. Moreover, the majority of confirmed malaria cases in Kampala (ranging from 61.8 to $81.0 \%$ in
2015 and 2018, respectively) were unaccounted for due to exclusion of facilities, leaving only up to $38 \%$ of the burden in this metropolitan district estimated (Table S1, Additional file 1). Excluding Kampala, however, results showed that estimates accounted for between 67 to $96 \%$ of the routine HMIS-based burden of malaria among the remaining 14 regions, over the study duration. Moreover, in these regions, average annual proportion of reported confirmed cases excluded from the study ranged from 5.3 to $19.8 \%$ in Karamoja and Tooro, respectively. Diagnostic testing of suspected malaria cases across the country was conducted either by microscopy or rapid diagnostic tests and reported as a single total.

\section{Mean incidence rates, seasonality, and risk of malaria}

The highest burden regions and districts also hosted health facilities with the highest number of confirmed malaria cases reported. For instance, Bala health centre (HC) III in Kole district of the Lango region reported 3317 cases during November 2015, while Bira HCII in Adjumani district of the West Nile region reported 6697 cases during June 2016. Moreover, Barakala HCIII (highest for two consecutive years) also from West Nile in Yumbe district, reported 9654 cases during October 2017 and 9246 cases during July 2018. Lastly, Matany hospital in Napak district of Karamoja region reported 8089 confirmed cases during September 2019.

This study showed spatial and temporal variation in incidence rates between regions and districts in any given region, as well as between health facility catchments within districts, both during the low (Fig. S8, Additional file 1) and high burden seasons (Fig. 2).

\section{National incidence rates}

The model estimated 38.8 (95\% CI: 37.9-40.9) million confirmed malaria cases over the study period of July, 2015 to September, 2019, highest in 2016 with 10.3 (95\% CI: 9.9-10.7) million cases and lowest in 2018 with 6.5 (95\% CI: 6.4-6.9) million cases among complete calendar years (Table S4, Additional file 1). Annual incidence rates reduced from 281.7 (95\% CI: 274.9-296.7) in 2016 to 170.0 (95\% CI: 165.9-178.8) cases per 1000 in 2018.

Monthly incidence rates showed a general declining trend in the burden of malaria from 2015 to 2019, strongest through 2018 followed by an increase in 2019 (Fig. 3). In all the years of the study, the incidence rates consistently peaked in June and July, reaching a maximum of 36.6 (95\% CI: 35.7-38.5) cases per 1000 in June 2017 (Table S5, Additional file 1), at regional (Fig. 3) and district (Fig. S11, Additional file 1) spatial scales. Conversely, low risk periods were less consistent, although often lowest in February and March, reaching a minimum of 8.9 (95\% CI: 8.7-9.4) in February 2018. 

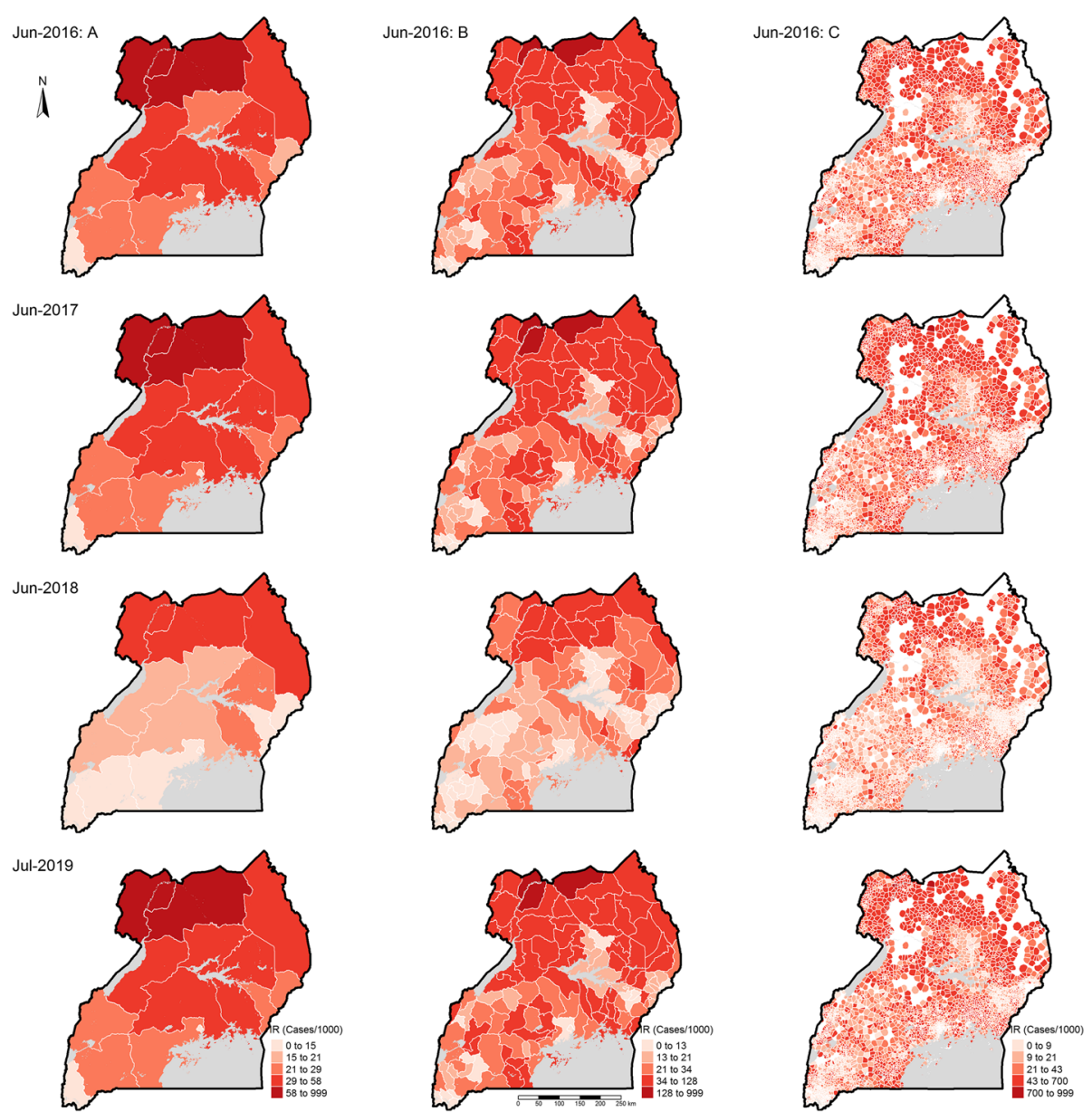

Fig. 2 Spatial distribution of malaria incidence rates during high burden months of study duration. Columns A, B, and C represent regions, districts, and heath facility catchments respectively, while the rows correspond to the respective highest burden month of each year. The lighter the shade of colour, the lower the incidence rates within a region, district, or catchment and the darker the colour, the higher the incidence rates

\section{Spatial distribution of incidence rates across the country}

Overall, mean monthly regional incidence rates were highest in Acholi region (Northern Uganda) at 52.3 (95\% CI: 50.3-59.6) cases per 1000 per month and lowest in Kigezi region (South Western Uganda) at 7.9 (95\% CI: 7.6-8.2) cases per 1000 per month (besides Kampala).

Consistent with national trend assessments, monthly trends in regional incidence rates showed the highest peaks in June-July, highest in June, 2017 (Range: 13.495.6 cases per 1000) and July, 2019 (Range: 13.5-95.5 cases per 1000 in Kigezi and Acholi, respectively) and the lowest troughs in February-March of each calendar year (Fig. 3). These trends showed that Acholi, West Nile, Karamoja, East Central - Busoga, and Teso persistently recorded the highest monthly incidence rates across the entire study duration. Moreover, the greatest variability in incidence rates was also observed among these five highest burden regions of with respective estimated mean monthly incidence rates of 52.3 (SD: 17.8),
43.3 (13.9), 30.3 (10.4), 26.3 (8.6), and 23.5 (8.0) cases per 1000 per month.

Within these regions, high burden and risk districts were also identified, both during the highest and lowest burden months. During June 2017 district monthly incidence reached the maximum in Lamwo of Acholi, Moyo of West Nile, Kaabong of Karamoja, Namayingo of East Central - Busoga, and Katakwi of Teso regions, at 167.6 (95\% CI: 165.6-169.8), 192.5 (95\% CI: 189.9-195.1), 81.1 (95\% CI: 79.6-82.5), 73.1 (95\% CI: 71.9-75.0), 72.0 (95\% CI: 70.9-73.1), cases per 1000 per month, respectively (Table S6, Additional file 1).

Monthly incidence rate trends among districts showed that Moyo, Lamwo, Adjumani, Pader, Nwoya, and Maracha persistently recorded the highest monthly incidence rates across the study duration (Fig. 3). Moreover, higher incidence rates were also associated with higher variability in monthly incidence rates with the mean monthly estimate in Moyo at 115.8 (SD: 36.5) and lower 


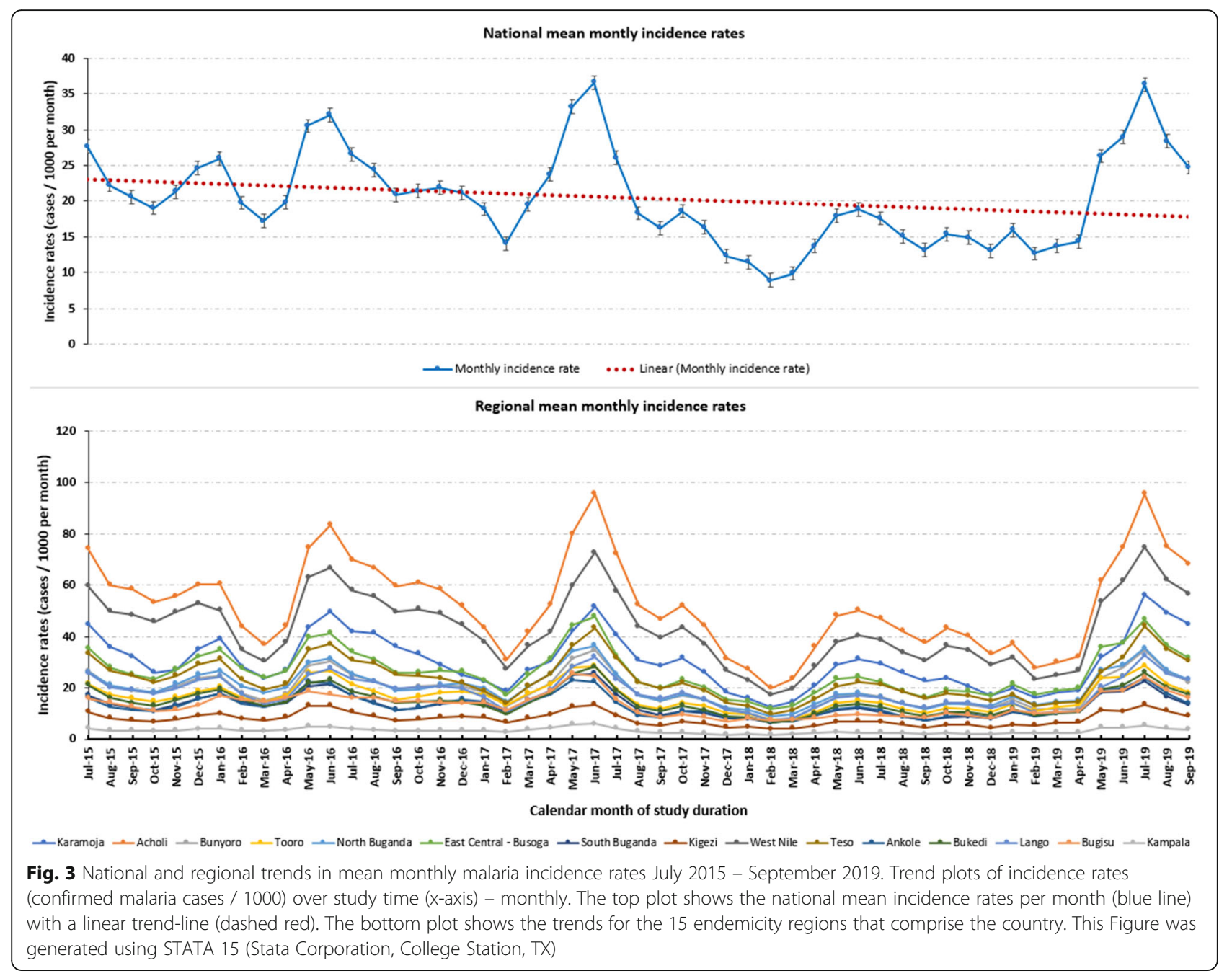

rates less variability with Rubanda at 1.6 (SD: 0.5) cases per 1000 (Figs. S10 and S11, Additional file 1).

Within individual districts, a wide distribution of incidence rates was estimated among health facility catchments both during the lowest and highest burden months. From the 3446 catchment areas identified across the country, mean monthly incidence rate reached a maximum of 569.8 (95\% CI: 555.2-584.3) cases per 1000 per month in Namayingo district of East Central - Busoga region and minimum of 0.13 (95\% CI: $0.10-0.17$ ) cases per 1000 per month in Rukungiri district of Kigezi region, excluding Kampala. Also, higher incidence rates within catchments were associated with higher viability in monthly incidence rates and lower incidence rates with less variability (Fig. S9, Additional file 1). Among health facility catchments, variability in incidence rates reached a maximum standard deviation $(\mathrm{SD})=142.4$ cases per 1000 in highest incidence rate catchment located in Namayingo and a minimum $\mathrm{SD}=0.1$ among the lowest burden catchments in Arua and Kasese districts.

\section{Spatial distribution of relative risk across the country}

Consistent with incidence rates, relative risk of malaria was highest among the highest burden regions of Acholi, West Nile, Karamoja, East Central - Busoga, and Teso, both during the lowest (Fig. S13, Additional file 1) and highest (Fig. S14, Additional file 1) burden months, maintaining their rank of risk at both times (Table S7, Additional file 1). During the highest burden month of June 2017, the relative risk of malaria among these regions ranged from 1.18 (95\% CI: $1.17-1.19$ ) to 2.6 (95\% CI: 2.6-2.8)-times higher than national average in Teso and Acholi, respectively. Moreover, while mean relative risk among districts within these regions was higher during the highest burden month at 1.8 (95\% Confidence Interval:1.5-2.1) than the lowest at 1.7 (95\% Conf. I:1.4-2.0), the difference was not significant $(p=0.676)$ by a two-sample t-test.

Spatial and temporal variation in relative risk observed between regions, and districts within regions (largely informative at programmatic or NMCP levels), was also 

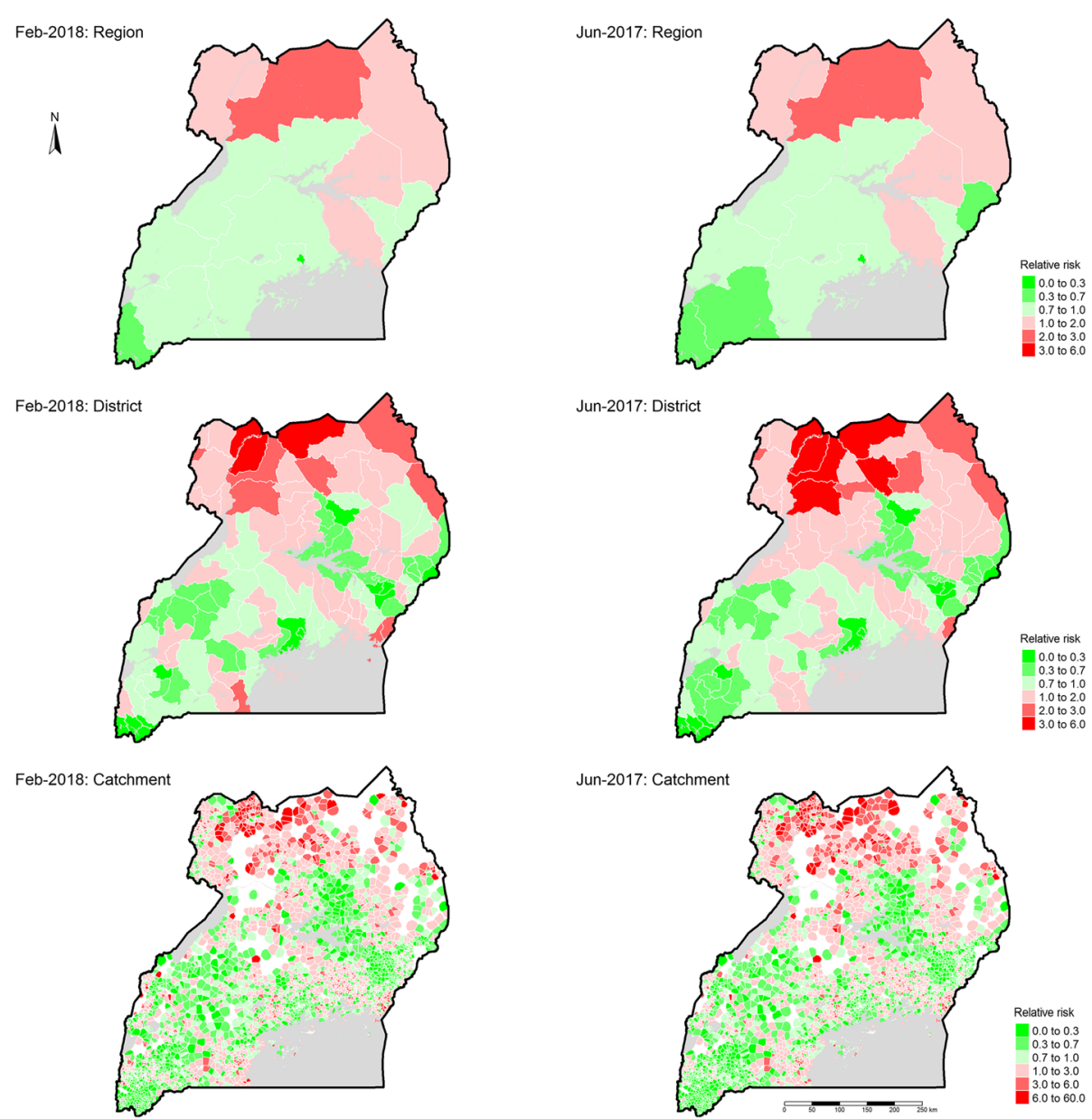

Fig. 4 Spatial distribution of the relative risk of malaria during lowest and highest burden months of the study duration. The left column shows, from top to bottom, relative risk by region, district, and health facility catchment for the lowest risk month of February 2018 while the right column shows a similar arrangement for the highest risk month of June 2017. For each row, the same levels (region, district, or health facility catchment) are side-by-side. Green areas are locations with relative risk of malaria lower than the national average where the darker the colour the lower levels of risk below national average. Red coloured areas are locations with relative risk of malaria higher than national average, where the darker the colour the high the risk

present between catchments within districts (informative for district health managers). Relative risk remained consistent among the 15 regions, between low and high burden seasons, but showed additional variability among districts and health facility catchments across the two seasons (Fig. 4).

Results showed that catchment risk ranged from 0 to 24.9 (95\% CI: 24.4-24.9) times higher than national average during the highest burden month and from 0 to 50.5 (95\% CI: $49.0-50.8$ ) during the lowest burden month. Moreover, a non-linear association of catchment risk was observed between the lowest and highest burden months further confirming this rising risk during lower burden months (Fig. S15, Additional file 1). However, the highest risk catchments at the two time points were neither identical nor located in the same district or region.

\section{Spatial clustering of risk}

Assessment for spatial autocorrelation of incidence and/or risk showed consistent levels of moderate global autocorrelation between both districts (Moran's I range by month: 0.4 to $0.6, p<0.001$ ) and health facility catchments $(0.3$ to $0.5, p<0.001)$. Both during the highest (June-2017) and lowest (February-2018) burden months, global autocorrelation between districts was very similar (Moran's $\mathrm{I}=0.5, p<0.001$ ) (Figs. 17 and 18, Additional file 1) but slight difference between health facility catchments (Moran's $\mathrm{I}=0.4$ and 0.3, $p<0.001$, respectively) (Figs. S19 and S20, Additional file 1).

Analysis of local spatial autocorrelation at two levels of significance ( $p<=0.05$ and $p<=0.01$ ) identified substantial significant high-high clustering in Acholi and West Nile regions in the North, as well as East Central - 


\section{Clusters}

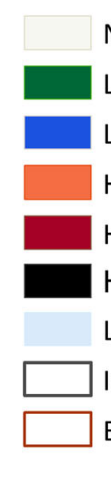

Not Significant

Low-Low Cluster

Low-High Outlier

High-Low Outlier

High-High Cluster $(p<=0.05)$

High-High Cluster $(p<=0.01)$

Lakes and rivers

International boundary

Endemicity regions
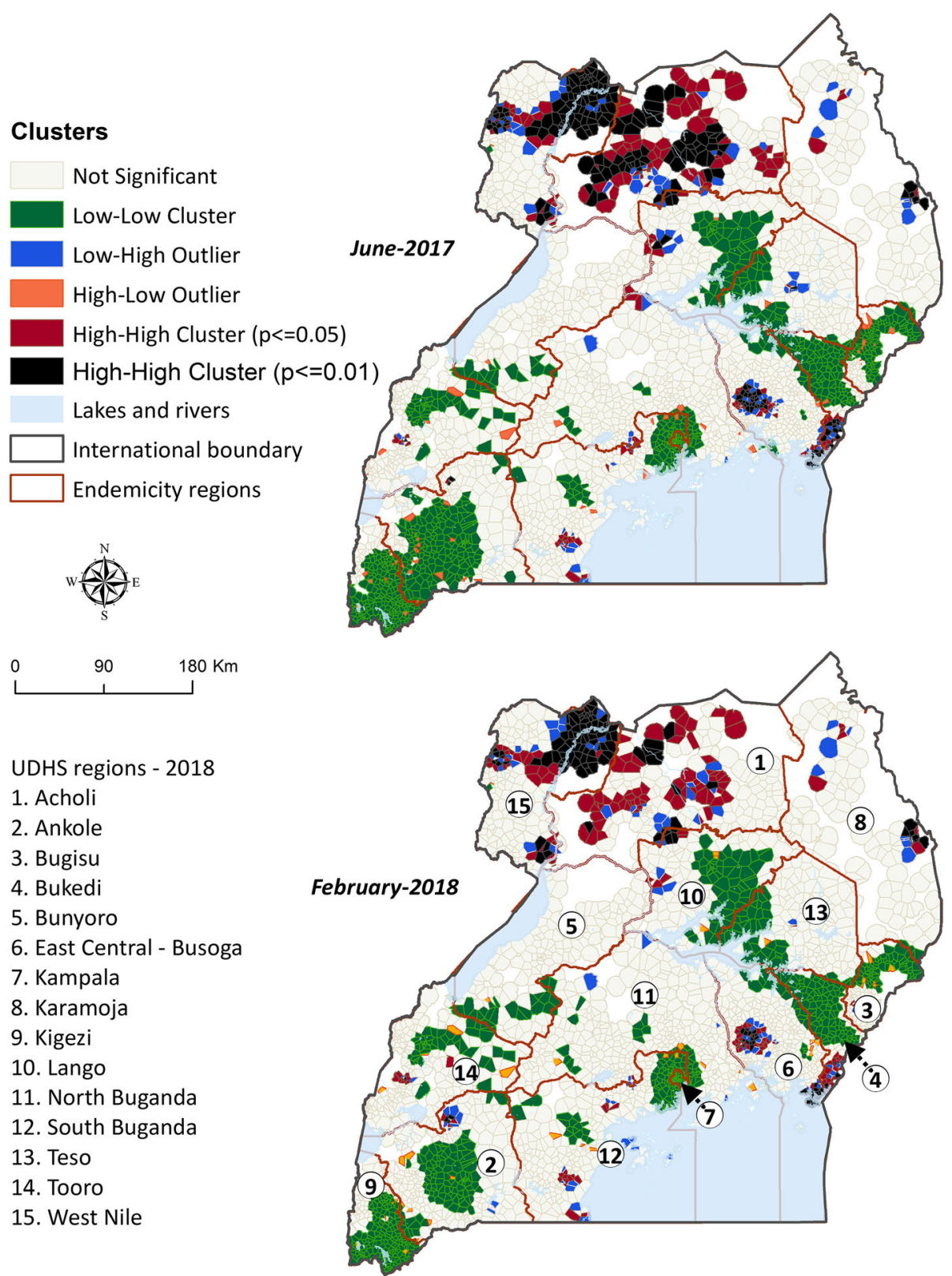

UDHS regions - 2018

1. Acholi

2. Ankole

3. Bugisu

4. Bukedi

5. Bunyoro

6. East Central - Busoga

7. Kampala

8. Karamoja

9. Kigezi

10. Lango

11. North Buganda

12. South Buganda

13. Teso

14. Tooro

15. West Nile

Fig. 5 Spatially significant clusters of malaria risk for the highest and lowest burden months between 2015 and 2019, across Uganda. The map at the top represents the distribution of significant clusters of malaria risk across the 15 regions of the country during the highest risk month of June 2017. The map at the bottom represents a similar distribution but for the lowest risk month of February 2018. High-High Clusters: The black and dark red areas represent the clusters of high-risk health facility catchments that are spatially located next to other high-risk catchments, with significant positive spatial autocorrelation at $<=0.01$ and $<=0.05$ levels of significance, respectively. This spatial autocorrelation was observed both during the lowest (Fig. S17, Additional file 1) and highest (Fig. S18, Additional file 1) burden seasons. High-Low Outliers: These orange areas represent high-risk clusters that are significantly disparate from their surrounding low-risk catchments. These outliers have significant negative spatial autocorrelation. Low-High Outliers: These blue areas represent the low-risk clusters that are significantly disparate from their surrounding high-risk catchments. These outliers also have significant negative spatial autocorrelation. Low-Low Clusters: These green areas represent the clusters of low-risk health facility catchments that are spatially located next to other low-risk catchments, with significant positive spatial autocorrelation. Not significant: The light grey areas represent the health facility catchments that did not show any significant spatial autocorrelation or clustering of either high, low or outlier distribution of risk of malaria. They are areas of highly random spatial distribution of risk of malaria 
Busoga region in the South East of the country, both during the highest and lowest burden seasons (Fig. 5). Similarly, large low-low clustering was identified in the Southern regions of the country. Moreover, outlier catchments typically had significantly lower risk than their neighbours in the north, and higher risk than their neighbours in the rest of the country. Significant monthly high-high clusters were comprised of between 191 health facility catchments during February 2018 and 236 during June 2017 and 2019 (Fig. S21, Additional file 1).

\section{Discussion}

Results from this innovative, large-scale, longitudinal observational study suggest that with improved HMIS reporting, credible high-risk areas at both high and low spatial scales were identifiable. The study revealed a distinct monthly spatial distribution of malaria incidence across the 15 regions of Uganda, in a concurrent multiresolution assessment, including coarse (regional) down to fine (health facility catchment) spatial resolutions. Moreover, whilst Uganda is considered a perennial transmission setting, this study revealed a nation-wide seasonal pattern in incidence rates with two peaks (major and minor), the highest during June-July and the minor peak during October. This approach may facilitate efficient implementation and optimization of targeted control activities that can leverage existing health facility systems [37]. It may also improve managers' understanding of the heterogeneity and/or clustering of malaria burden within districts that currently form the lowest level of malaria burden assessments, though acknowledged as difficult to use or unusable for control planning [5].

This study showed that the risk of malaria by regional rank, among the highest and lowest risk regions, had minimal temporal variability. These regions maintained their status both during low and high burden seasons. These findings were consistent with extant UDHS regional stratification of Uganda where Acholi, West Nile, and Karamoja were among the highest transmission regions, and Ankole and Kigezi among the lowest. This stratification supports tailored approaches for long-term malaria control efforts aiming at elimination, as advocated in the global 'high burden to high impact' initiative [17] that was recently adopted as central to onward national malaria control strategies for Uganda [38]. Whilst targeted interventions, including IRS [39] and larval source management [40] have been used, further emphasis is necessary $[17,41]$ with implementation taking greater account of local context. Importantly, however, temporal variability of risk among many regions highlights the continued vital role of routine surveillance for planning and timely action towards control.
Moreover, higher risk among high burden locations during the lowest than highest burden seasons suggests persistent high-risk in these locations, the identification of which could facilitate high precision targeted actions for effective control.

This study also identified several distinct clusters of high-risk health facility catchments, which were consistent over time though largest during the highest burden seasons and smallest at the lowest. The largest high-risk clusters were concentrated in the West Nile and Acholi regions in Northern Uganda, although smaller clusters were noted in the recognised high transmission regions of Karamoja and East-Central Busoga [19]. Conversely, the most notable low-risk health facility catchment clusters could be grouped into three categories: highland regions (e.g. Kigezi, Ankole and Bugisu) [42, 43]; regions with recent intense targeted multi-year IRS activity associated with high impacts on transmission (e.g. Bukedi, Teso, and Lango) $[4,19,44,45]$; and, large urban municipalities (e.g. Southern Buganda) with urbanization associated with reduced transmission [46, 47]. These findings provide further evidence of identifiable candidate locations for targeted control interventions among the high-risk clusters and an approach for assessment of possible impacts of previous interventions.

Trends in annual confirmed malaria cases in Uganda declined between 2016 and 2018, despite increased reporting and proportions of confirmed cases over time, consistent with MIS findings between 2014 and 2018 [4, 19], before a sharp increase in 2019. Moreover, the relationship between regional relative risk and prevalence of malaria (among children under 5 years of age from the 2018 MIS) showed that small changes in parasite prevalence were associated with sharp increases in relative risk among regions at lower than national average risk. However, large changes in parasite prevalence were associated with small changes in relative risk among regions at higher than national average risk. This further confirms the variability of risk among many regions while pointing to strong effects of age on malaria [48]. Besides the estimated confirmed cases being lower than estimates reported by WHO and MAP per year (possibly due to study design of excluding some facilities), trends were also dissimilar with WHO and MAP cases increasing between 2016 and 2017 [18], unlike in this present study. Nevertheless, such dissimilarities have been documented [3] and are likely explained by the use in global assessment for sub-Saharan Africa of prevalence surveys that to date, are predominantly conducted among children [49]. With estimates for the whole population generated from these surveys, despite shifts in malaria burden from children to the older population following effective control interventions 
[48], the dynamic effects on burden may not be adequately accounted for in the prevalence-toincidence models used.

The observed seasonality with June-July peaks and February-March troughs was consistent with reports from south western Uganda, where epidemics followed a regular July pattern except during El-nino in 1998 $[5,50]$ and in Gulu district (Northern Uganda) where between 2006 and 2015 biannual peaks of malaria were reported during June-July and October-November [51]. One study however, reported two peaks of malaria during April-May and September-November in Northern Uganda following the rain seasons, though unsubstantiated [52]. Findings from this present study may inform optimal timing for control activities, including IRS, mass drug administration (MDA), or community mobilization campaigns towards increased malaria risk awareness for control vigilance.

PFP facilities, a small majority of which do not report to the HMIS and were therefore excluded from this study, limit the utility of focal analyses such as presented here. This highlights an important missed surveillance opportunity. The limited capacity to detect outbreaks in settings largely served by PFP may exacerbate the severity of malaria outcomes among their most vulnerable residents, coupled with increased case management costs [53]. There are several possible initiatives to increase reporting in these facilities where a small majority seek care for febrile illnesses [4, 19, 54]. First, provision of guarantees on exclusive use of data for public health not revenue monitoring, may improve confidence and alleviate any fears of punitive intensions in their reporting. Second, ensured availability of standardized reporting tools, may offset running costs of stationery in the private facilities while it enables improved documentation of health records. Third, training of PFP managers and owners on the benefits of surveillance and/or reporting may increase their involvement. Lastly, implementation of regular feedback mechanisms may provide a means of continued evaluation that fosters risk and other assessments that are mutually beneficial.

Given that policymakers' remediating responses as well as policy formulation processes are informed by pooled information from diverse sources, including but not limited to research, political, and funding provisions, it is unrealistic to expect these technocrats to be expert generators of the evidence from these multi-disciplinary sources. Whilst there are no simple solutions to the implementation of analyses such as in this present study, interpretation of contemporary outputs is nowhere nearly as demanding, highlighting the criticality of partnerships between policy and research dimensions for malaria and other disease control efforts.
This study had limitations. First, the disproportionately low proportion of geolocated reporting private facilities impacted on the estimates of malaria burden, especially among highly urban locations, including Kampala and Wakiso districts and others across the country. Results for the Kampala region (and Wakiso district) in this study, represent only a small proportion of the burden and were excluded from results discussions. Moreover, exclusion of non-geolocated reporting public health facilities (such as in Kitgum district), impacted on the estimates of incidence due to unidentified catchments in those places. Nevertheless, there was wide coverage of health facilities across the country with a small proportion of districts under-represented, minimizing effects of this constraint. Second, the study did not account for level of health facility and other population level factors that impact on differential health seeking behaviour, which may have inflated incidence rates and risk, where a given level or type of facility is preferred. However, in this analysis it was assumed that for uncomplicated malaria, people attend the closest health facility and some important factors such as urbanicity and primary care giver education were accounted for, though further research may be required to better understand impacts of level of health facility on care seeking for uncomplicated malaria. Third, the study did not account for stock levels of antimalarials or test kits, variations of which may impact on the number of cases recorded between seasons of full stock versus stockouts. A better understanding of the linkage between logistics management and HMIS may be required, given known associations between stockouts and increased under-five mortality or compromised treatment practices like dosage rationing and use of less effective remedies [55]. Fourth, given that health facility recruitment into the study was not dynamic, any increase in number of facilities reporting could have had impacts on study findings. Moreover, the systematic exclusion of non-geolocated facilities, may have biased study results towards more long-term established than newer health facilities, but duration of facility existence was beyond the scope of this study.

\section{Conclusion}

Assessment of malaria burden and/or risk in high burden countries using routine surveillance data is highly achievable. Using national routine data, this study provided needed evidence of vital concurrent assessment of malaria risk and burden among regions, districts, and health facility catchments with identifiable significant spatial clustering of risk. Targeting hotspots as an intervention approach has been shown to yield modest and transient impacts on malaria prevalence [56]. However, locations with persistently high-risk of malaria that are potential candidates for health facility-based interventions such as 
community outreaches, provision of LLINs, mass drug administration and enhanced case management were identified, an approach that may be beneficial beyond isolated health facility catchments. Furthermore, whilst extensive geo-spatial analytical output with scales either too large (region or district) or too fine (pixel or neighbourhood) may be challenging for control programmes to use [57], this study provides evidence of HMIS-based assessments at practical scales for districts to implement and assess intervention impacts. Moreover, in perennial settings, the identifiable strong seasonal patterns as seen with June-July highest peaks and February-March lowest troughs in Uganda, provide vital information for intervention timing. Taken together, these results show the potential in routine HMIS surveillance data for pragmatic timely identification of high-risk areas and with further research, the assessments for optimal implementation of targeted control activities and their impacts.

\section{Supplementary Information}

The online version contains supplementary material available at https://doi. org/10.1186/s12889-020-10007-w.

\section{Additional file 1.}

\section{Abbreviations}

ACTs: Artemisinin-based combination therapies; AL: Artemether-lumefantrine; B/S: Blood slide microscopy; BYM: Besag, York and Mollie modelling approach; Cl: Credible interval; DEM: Digital elevation model; DHIS-2: District health information system - version 2; DHS: Demographic health survey; HMIS: Health management information system; INLA: Integrated nested Laplace approximation; IRS: Indoor residual spraying with insecticide; KEMRI: Kenya Medical Research Institute; LLIN: Long-lasting insecticidal net; MAP: Malaria Atlas Project; MIS: Malaria indicator survey; $\mathrm{MoH}$ : Uganda Ministry of Health; NDVI: Normalized differences vegetation index; NMCP: National malaria control programme; OPD: Outpatient department; PFP: Private for profit; PNFP: Private not for profit; RDT: Rapid diagnostic test for malaria; UBOS: Uganda bureau of statistics; UTM: Universal Transverse Mercator coordinate system; WGS: World Geodetic System of modelling earth; WHO: World Health Organization

\section{Acknowledgements}

We would like to thank Katonda Omukulu for his support for this project. We would also like to acknowledge Deborah Ekusai, Rhoda Namubiru, Faith Bagatya, Dr. Charles Olara, Dr. Joaniter Nankabirwa and the administration and staff at the National Malaria Control Division in charge of the national HMIS and the DHIS-2 reporting for their contributions, as well as the administration and staff at the Infectious Diseases Research Collaboration and the Makerere University College of health sciences for their support.

\section{Authors' contributions}

SPK and RP conceived of the study. SPK led the data collation with support from RK, JC, CMS, DR, and JO. SPK led the data analysis with support from BS and RP. SPK led the manuscript drafting with support from $T B, A Y, A G, B S$, and RP. SPK and BS had full access to all the data in this study, but all authors reviewed the manuscript and gave permission for publication. SPK, the corresponding author, had final responsibility for the decision to submit for publication. The author(s) read and approved the final manuscript.

\section{Funding}

Funding for the research reported in this publication was supported by the Fogarty International Center of the National Institutes of Health under Award Numbers (D43TW7375) and (D43TW010526). The content is solely the responsibility of the authors and does not necessarily represent the official view of the National Institutes of Health.

\section{Availability of data and materials}

The datasets used and/or analyzed for this study are available from the corresponding author on reasonable request and with permission from the Department of Health Information, Uganda Ministry of Health.

\section{Ethics approval and consent to participate}

This study was approved by the Uganda National Council for Science and Technology (UNCST Ref SS 4455), the Uganda Ministry of Health (ADM.386/ 01), the Makerere University School of Medicine Research and Ethics Committee (REC Ref. 2017-119), and the London School of Hygiene \& Tropical Medicine Ethics Committee (LSHTM Ref. 13902-1).

Written informed consent of participants included in this study was not applicable.

\section{Consent for publication}

Not applicable.

\section{Competing interests}

The authors declare that they have no competing interests.

\section{Author details}

${ }^{1}$ Department of Disease Control, London School of Hygiene \& Tropical Medicine, Keppel Street, London WC1E 7HT, UK. ${ }^{2}$ Infectious Diseases Research Collaboration, PO Box 7475, Kampala, Uganda. ${ }^{3}$ USAID's Malaria Action Program for Districts, PO Box 8045, Kampala, Uganda. ${ }^{4}$ National Malaria Control Division, Uganda Ministry of Health, Kampala, Uganda. ${ }^{5}$ Department of Medical Microbiology, Radboud University, Nijmegen, Netherlands. ${ }^{6}$ Department of Disease Control and Environmental Health, College of Health Sciences, School of Public Health, Makerere University, PO Box 7072, Kampala, Uganda. ${ }^{7}$ African Leaders Malaria Alliance (ALMA), Kampala, Uganda.

Received: 7 August 2020 Accepted: 4 December 2020

Published online: 14 December 2020

\section{References}

1. Bhatt S, Weiss DJ, Cameron E, Bisanzio D, Mappin B, Dalrymple U, Battle KE, Moyes $C L$, Henry A, Eckhoff PA, et al. The effect of malaria control on plasmodium falciparum in Africa between 2000 and 2015. Nature. 2015;526: 207-11.

2. WHO. World Malaria Report 2015. Geneva: World Health Organization; 2015

3. WHO. World Malaria Report 2018. Geneva: World Health Organization; 2018.

4. International UBoSUal. Uganda malaria Indicator survey 2014-15. Kampala and Rockville: UBOS and ICF International; 2015.

5. Talisuna AO, Noor AM, Okui AP, Snow RW. The past, present and future use of epidemiological intelligence to plan malaria vector control and parasite prevention in Uganda. Malar J. 2015;14:158.

6. Nisingizwe MP, lyer HS, Gashayija M, Hirschhorn LR, Amoroso C, Wilson R, Rubyutsa E, Gaju E, Basinga P, Muhire A, et al. Toward utilization of data for program management and evaluation: quality assessment of five years of health management information system data in Rwanda. Glob Health Action. 2014;7:25829.

7. Wagenaar BH, Gimbel S, Hoek R, Pfeiffer J, Michel C, Manuel JL, Cuembelo F, Quembo T, Afonso P, Porthe $V$, et al. Effects of a health information system data quality intervention on concordance in Mozambique: time-series analyses from 2009-2012. Popul Health Metrics. 2015;13:9.

8. WHO. Global technical strategy for malaria 2016-2030. Geneva: World Health Organization; 2015.

9. Maokola W, Willey BA, Shirima K, Chemba M, Armstrong Schellenberg JR, Mshinda H, Alonso P, Tanner M, Schellenberg D. Enhancing the routine health information system in rural southern Tanzania: successes, challenges and lessons learned. Tropical Med Int Health. 2011;16:721-30.

10. Mate KS, Bennett B, Mphatswe W, Barker P, Rollins N. Challenges for routine health system data management in a large public programme to prevent mother-to-child HIV transmission in South Africa. PLoS One. 2009:4:e5483.

11. Ploubidis GB, Palmer MJ, Blackmore C, Lim TA, Manissero D, Sandgren A, Semenza JC. Social determinants of tuberculosis in Europe: a prospective ecological study. Eur Respir J. 2012;40:925-30. 
12. P. C. Representing spatial dependence and spatial discontinuity in ecological epidemiology: a scale mixture approach. Stoch Env Res Risk A. 2016;31:291-304

13. Okami S, Kohtake N. Spatiotemporal modeling for fine-scale maps of regional malaria endemicity and its implications for transitional complexities in a routine surveillance network in Western Cambodia. Front Public Health. 2017;5:262.

14. Rodriguez-Rodriguez D, Maraga S, Jamea-Maiasa S, Tandrapah A, Makita L, Siba PM, Mueller I, Pulford J, Hetzel M. Mapping routine malaria incidence at village level for targeted control in Papua New Guinea. Geospat Health. 2019;14.

15. Mlacha YP, Chaki PP, Malishee AD, Mwakalinga VM, Govella NJ, Limwagu AJ, Paliga JM, Msellemu DF, Mageni ZD, Terlouw DJ, et al. Fine scale mapping of malaria infection clusters by using routinely collected health facility data in urban Dar es Salaam, Tanzania. Geospat Health. 2017:12:494.

16. Ver Hoef JM, Peterson EE, Hooten MB, Hanks EM, Fortin M. Spatial autoregressive models for statistical inference from ecological data. Ecol Soc Am. 2017:88:36-59.

17. $\mathrm{WHO}, \mathrm{RBM}$. High burden to high impact: a targeted malaria response. Geneva: World Health Organization; 2019.

18. WHO. World Malaria Report 2019. Geneva: World Health Organization; 2019.

19. (NMCD) UNMCD, (UBOS) UBoS, ICF. Uganda Malaria Indicator Survey 201819. Kampala and Rockville: NMCD, UBOS, and ICF; 2020.

20. Kiberu VM, Matovu JK, Makumbi F, Kyozira C, Mukooyo E, Wanyenze RK Strengthening district-based health reporting through the district health management information software system: the Ugandan experience. BMC Med Inform Decis Mak. 2014;14:40.

21. Graetz N, Friedman J, Osgood-Zimmerman A, Burstein R, Biehl MH, Shields C, Mosser JF, Casey DC, Deshpande A, Earl L, et al. Mapping local variation in educational attainment across Africa. Nature. 2018;555:48-53.

22. Awor $P$, Wamani $H$, Bwire $G$, Jagoe $G$, Peterson S. Private sector drug shops in integrated community case management of malaria, pneumonia, and diarrhea in children in Uganda. Am J Trop Med Hyg. 2012;87:92-6.

23. Cohen J, Cox A, Dickens W, Maloney K, Lam F, Fink G. Determinants of malaria diagnostic uptake in the retail sector: qualitative analysis from focus groups in Uganda. Malar J. 2015;14:89.

24. Ray N, Ebener S. AccessMod 3.0: computing geographic coverage and accessibility to health care services using anisotropic movement of patients. Int J Health Geogr. 2008;7:63.

25. Alegana VA, Wright JA, Pentrina U, Noor AM, Snow RW, Atkinson PM. Spatial modelling of healthcare utilisation for treatment of fever in Namibia. Int J Health Geogr. 2012;11:6.

26. Aoun N, Matsuda H, Sekiyama M. Geographical accessibility to healthcare and malnutrition in Rwanda. Soc Sci Med. 2015;130:135-45.

27. Ouma PO, Maina J, Thuranira PN, Macharia PM, Alegana VA, English M, Okiro EA, Snow RW. Access to emergency hospital care provided by the public sector in sub-Saharan Africa in 2015: a geocoded inventory and spatial analysis. Lancet Glob Health. 2018:6:e342-50.

28. Lee D. A comparison of conditional autoregressive models used in Bayesian disease mapping. Spat Spatiotemporal Epidemiol. 2011;2:79-89.

29. Riebler A, Sorbye SH, Simpson D, Rue H. An intuitive Bayesian spatial model for disease mapping that accounts for scaling. Stat Methods Med Res. 2016; 25:1145-65.

30. Goovaerts $P$, Gebreab S. How does Poisson kriging compare to the popular BYM model for mapping disease risks? Int J Health Geogr. 2008;7:6.

31. Team RC. R: A language and environment for statistical computing. Vienna: R Foundation for Statistical Computing; 2018.

32. Bernardinelli $L$, Clayton D, Montomoli C. Bayesian estimates of disease maps: how important are priors? Stat Med. 1995;14:2411-31.

33. Gething PW, Patil AP, Smith DL, Guerra CA, Elyazar IR, Johnston GL, Tatem AJ, Hay SI. A new world malaria map: plasmodium falciparum endemicity in 2010. Malar J. 2011;10:378.

34. Nihei $N$, Hashida Y, Kobayashi M, Ishii A. Analysis of malaria endemic areas on the Indochina peninsula using remote sensing. Jpn J Infect Dis. 2002;55: 160-6.

35. Cohen JM, Ernst KC, Lindblade KA, Vulule JM, John CC, Wilson ML. Local topographic wetness indices predict household malaria risk better than land-use and land-cover in the western Kenya highlands. Malar J. 2010;9:328.

36. WHO. World Malaria Report 2017. Geneva: World Health Organization; 2017.

37. J P, F G, J-M C, HB Mn, J-F J. Analysing Spatial-Temporal Clustering of Meningococcal Meningitis Outbreaks in Niger Reveals Opportunities for Improved Disease Control. PLoS Negl Trop Dis. 2012;6:e1577.
38. $\mathrm{MOH}$. Uganda becomes the first country to launch the high burden high impact response: Ministry of Health; 2019.

39. Kigozi R, Baxi SM, Gasasira A, Sserwanga A, Kakeeto S, Nasr S, Rubahika D, Dissanayake G, Kamya MR, Filler S, Dorsey G. Indoor residual spraying of insecticide and malaria morbidity in a high transmission intensity area of Uganda. PLoS One. 2012;7:e42857.

40. MoH. Annual health sector performance report 2018/2019. Kampala: Uganda Ministry of Health; 2019.

41. WHO. WHO Technical brief for countries preparing malaria funding requests for the Global Fund (2020-2022). Geneva: World Health Organization; 2020.

42. Bodker R, Akida J, Shayo D, Kisinza W, Msangeni HA, Pedersen EM, Lindsay SW. Relationship between altitude and intensity of malaria transmission in the Usambara Mountains, Tanzania. J Med Entomol. 2003:40:706-17.

43. Siraj AS, Santos-Vega M, Bouma MJ, Yadeta D, Ruiz Carrascal D, Pascual M. Altitudinal changes in malaria incidence in highlands of Ethiopia and Colombia. Science. 2014;343:1154-8.

44. Steinhardt LC, Yeka A, Nasr S, Wiegand RE, Rubahika D, Sserwanga A, Wanzira H, Lavoy G, Kamya M, Dorsey G, Filler S. The effect of indoor residual spraying on malaria and anemia in a high-transmission area of northern Uganda. Am J Trop Med Hyg. 2013;88:855-61.

45. Tugume A, Muneza F, Oporia F, Kiconco A, Kihembo C, Kisakye AN, Nsubuga P, Deogratias S, Yeka A. Effects and factors associated with indoor residual spraying with Actellic 300 CS on malaria morbidity in Lira District, Northern Uganda. Malar J. 2019;18:44

46. Hay SI, Guerra CA, Tatem AJ, Atkinson PM, Snow RW. Urbanization, malaria transmission and disease burden in Africa. Nat Rev Microbiol. 2005:3:81-90.

47. Kabaria CW, Gilbert M, Noor AM, Snow RW, Linard C. The impact of urbanization and population density on childhood plasmodium falciparum parasite prevalence rates in Africa. Malar J. 2017;16:49.

48. Kigozi SP, Kigozi RN, Epstein A, Mpimbaza A, Sserwanga A, Yeka A, Nankabirwa Jl, Halliday K, Pullan RL, Rutazaana D, et al. Rapid shifts in the age-specific burden of malaria following successful control interventions in four regions of Uganda. Malar J. 2020:19:128.

49. Guerra CA, Hay SI, Lucioparedes LS, Gikandi PW, Tatem AJ, Noor AM, Snow RW. Assembling a global database of malaria parasite prevalence for the malaria atlas project. Malar J. 2007;6:17.

50. Mouchet J, Manguin S, Sircoulon J, Laventure S, Faye O, Onapa AW, Carnevale P, Julvez J, Fontenille D. Evolution of malaria in Africa for the past 40 years: impact of climatic and human factors. J Am Mosq Control Assoc. 1998;14:121-30

51. Simple O, Mindra A, Obai G, Ovuga E, Odongo-Aginya El. Influence of climatic factors on malaria epidemic in Gulu District, northern Uganda: a 10year retrospective study. Malar Res Treat. 2018;2018:5482136.

52. Tukei BB, Beke A, Lamadrid-Figueroa H. Assessing the effect of indoor residual spraying (IRS) on malaria morbidity in northern Uganda: a before and after study. Malar J. 2017:16:4.

53. Keiser J, Utzinger J, Caldas de Castro M, Smith TA, Tanner M, Singer BH. Urbanization in sub-saharan Africa and implication for malaria control. Am J Trop Med Hyg. 2004;71:118-27.

54. Macro UBoSUal. Uganda malaria Indicator survey 2009. Calverton: UBOS and ICF Macro; 2010

55. Hamel MJ, Adazu K, Obor D, Sewe M, Vulule J, Williamson JM, Slutsker L, Feikin DR, Laserson KF. A reversal in reductions of child mortality in western Kenya, 2003-2009. Am J Trop Med Hyg. 2011;85:597-605.

56. Bousema T, Stresman G, Baidjoe AY, Bradley J, Knight P, Stone W, Osoti V, Makori E, Owaga C, Odongo W, et al. The impact of hotspot-targeted interventions on malaria transmission in Rachuonyo South District in the Western Kenyan highlands: a cluster-randomized controlled trial. PLoS Med. 2016:13:e1001993.

57. Tatem AJ. Innovation to impact in spatial epidemiology. BMC Med. 2018;16: 209.

\section{Publisher's Note}

Springer Nature remains neutral with regard to jurisdictional claims in published maps and institutional affiliations. 